\title{
The abundance of $\mathrm{HOC}^{+}$in diffuse clouds
}

\author{
H. Liszt ${ }^{1}$, R. Lucas ${ }^{2}$, and J. H. Black ${ }^{3}$ \\ 1 National Radio Astronomy Observatory, 520 Edgemont Road, Charlottesville, VA 22903-2475, USA \\ e-mail: hliszt@nrao.edu \\ 2 Institut de Radioastronomie Millimétrique, 300 rue de la Piscine, 38406 Saint-Martin-d'Hères, France \\ 3 Chalmers Centre for Astrophysics and Space Science, Onsala Space Observatory, Chalmers University of Technology, \\ 43992 Onsala, Sweden
}

Received 12 July 2004 / Accepted 23 August 2004

\begin{abstract}
We used the Plateau de Bure Interferometer to search for $\lambda 3 \mathrm{~mm}$ absorption lines of $\mathrm{HOC}^{+}$from local diffuse and translucent clouds occulting compact extragalactic mm-wave continuum sources. We detected $\mathrm{HOC}^{+}$in three directions with column densities only 70-120 times below that of the $\mathrm{HCO}^{+}$isomer, a factor 5-50 higher than typically found in dense dark gas but comparable to recent observations of dense photon-dominated regions. The observed amounts of $\mathrm{HOC}^{+} N\left(\mathrm{HOC}^{+}\right) / N\left(\mathrm{H}_{2}\right)=$ $3-6 \times 10^{-11}$ can be made in quiescent diffuse gas at thermal gas-kinetic rates if the $\mathrm{H}_{2} \mathrm{O} / \mathrm{OH}$ ratio is of order unity, in mild violation of extant observational limits.
\end{abstract}

Key words. ISM: molecules - astrochemistry

\section{Introduction}

The roster of polyatomic molecules known to exist in diffuse interstellar gas has been steadily enlarged over the last fifteen years and now numbers nearly one dozen. Two of these, $\mathrm{C}_{3}$ (Maier et al. 2001; Roueff et al. 2002; Oka et al. 2003) and $\mathrm{H}_{3}{ }^{+}$(McCall et al. 2003) were recently discovered in optical and near-infrared absorption spectra. The others have accumulated gradually over the last twenty or so years in cm-wave and $\mathrm{mm}$-wave radiofrequency absorption data and now include $\mathrm{H}_{2} \mathrm{CO}$ (Federman \& Willson 1982; Nash 1990; Marscher et al. 1993; Liszt \& Lucas 1995; Moore \& Marscher 1995), $\mathrm{HCO}^{+}$ (Lucas \& Liszt 1996), $\mathrm{NH}_{3}$ (Nash 1990), $\mathrm{C}_{2} \mathrm{H}$ (Lucas \& Liszt 2000), $\mathrm{C}_{3} \mathrm{H}_{2}$ (Cox et al. 1988; Lucas \& Liszt 2000), HCN and $\mathrm{HNC}$ (Liszt \& Lucas 2001) and $\mathrm{H}_{2} \mathrm{~S}$ and $\mathrm{HCS}^{+}$(Lucas \& Liszt 2002).

Observationally, the polyatomics observed with radio techniques are often seen to be strongly and directly related to simpler species observed at optical wavelengths, i.e. $\mathrm{HCO}^{+}$to OH (Liszt \& Lucas 1996; Lucas \& Liszt 1996) HCN and HNC to $\mathrm{CN}$ (Liszt \& Lucas 2001) etc. These connections point to fundamental lacunae in our understanding of the chemistry of even the simplest species in diffuse clouds: simple models which reproduce the abundances of diatomics, however easily, invariably fall orders of magnitude short of explaining the related polyatomics. Conversely, the high abundances of some polyatomics transform other mysteries; the poorly-understood abundances of CO and CS in diffuse clouds are readily understandable given the recombination of the observed columns of $\mathrm{HCO}^{+}$(Liszt \& Lucas 2000) and $\mathrm{HCS}^{+}$(Lucas \& Liszt 2002) respectively.
The work described here rounds out to an even dozen the number of polyatomics known to exist in diffuse and translucent clouds. $\mathrm{HOC}^{+}$, the energetically-disfavored $\mathrm{HCO}^{+}$isomer whose $89.4874 \mathrm{GHz} J=1-0$ transition we sought, was indeed detected along several of the sightlines studied in the other molecular species mentioned in the preceding paragraphs. The $\mathrm{HCO}^{+}-\mathrm{HOC}^{+}$comparison is especially interesting now because both species have recently been detected over a wide range of physical conditions in dense gas: our new spectra reinforce an emerging view which relates molecular abundances in dense and diffuse PDR. Section 2 gives details of the observations, which are discussed in Sect. 3 and summarized in Sect. 4.

\section{Observations of $\mathrm{HOC}^{+}$}

The $89.4874 \mathrm{GHz} J=1-0$ transition of $\mathrm{HOC}^{+}$was observed at the Plateau de Bure at various times during the period 2000-2001 toward the five sources listed in Table 1. Profiles were taken with $39.1 \mathrm{kHz}\left(0.13 \mathrm{~km} \mathrm{~s}^{-1}\right)$ channel separation and $70 \mathrm{kHz}$ resolution and subsequently once hanning smoothed, yielding spectra with the line/continuum rms values as listed in Table 1.

The column density in the lowest $(J=0)$ level of a simple linear molecule is related to the integrated optical depth of the $J=1-0$ transition as

$N_{0}=\frac{8.0 \times 10^{12} \mathrm{~cm}^{-2} \int \tau_{10} \mathrm{~d} v}{\mu^{2}\left(1-\exp \left(-h v_{10} / k T_{\text {exc }}\right)\right)}$ 
Table 1. Background sources observed.

\begin{tabular}{lcccc}
\hline \hline Source & $l$ & $b$ & $\sigma_{1 / \mathrm{c}}^{1}$ & $\int \tau\left(\mathrm{HOC}^{+}\right) \mathrm{d} v$ \\
\hline & & & & $\mathrm{km} \mathrm{s}^{-1}$ \\
\hline B0355+508 & $150.38^{\circ}$ & $-1.60^{\circ}$ & 0.0022 & $0.042(0.005)$ \\
B0415+379 & $161.68^{\circ}$ & $-8.82^{\circ}$ & 0.0029 & $0.051(0.004)$ \\
B0528+134 & $191.37^{\circ}$ & $-11.01^{\circ}$ & 0.0101 & $<0.036$ \\
B1730-130 & $12.03^{\circ}$ & $+10.81^{\circ}$ & 0.0081 & $<0.026$ \\
B2200+420 & $92.13^{\circ}$ & $-10.40^{\circ}$ & 0.0041 & $0.017(0.006)$ \\
\hline
\end{tabular}

${ }^{1} \mathrm{rms}$ noise in line/continuum ratio at $140 \mathrm{kHz}\left(0.46 \mathrm{~km} \mathrm{~s}^{-1}\right)$ resolution.

Table 2. Column densities.

\begin{tabular}{lccc}
\hline \hline Source & $N\left(\mathrm{HOC}^{+}\right)$ & $N\left(\mathrm{HCO}^{+}\right)$ & $N(\mathrm{OH})$ \\
& $10^{10} \mathrm{~cm}^{-2}$ & $10^{12} \mathrm{~cm}^{-2}$ & $10^{13} \mathrm{~cm}^{-2}$ \\
\hline B0355+508 & $9.01(1.07)$ & $6.51(0.02)$ & $14.87(0.67)$ \\
B0415+379 & $10.9(0.90)$ & $12.8(0.40)^{2}$ & $42.50(0.10)$ \\
B0528+134 & $<7.7^{1}$ & $2.28(0.02)$ & $4.72(0.31)$ \\
B1730-130 & $<5.7^{1}$ & $1.25(0.02)$ & $1.34(0.08)$ \\
B2200+420 & $3.7(1.3)$ & $2.58(0.03)$ & $5.73(0.21)$ \\
\hline
\end{tabular}

${ }^{1}$ Limits are $2 \sigma$.

${ }^{2}$ Entry is $59 \pm 2 N\left(\mathrm{H}^{13} \mathrm{CO}^{+}\right)$.

where $\mu$ is the permanent dipole moment of the molecule, $T_{\text {exc }}$ is the excitation temperature of the $J=1-0$ transition and $v_{10}$ is its frequency.

With the exception of CO (Liszt \& Lucas 1998), the molecules observed at $\mathrm{mm}$-wavelengths in diffuse gas are well-described by assuming that the rotational ladder is thermalized at the temperature of the cosmic microwave background, in which case the total column density can be calculated straightforwardly from observations of one transition. We take the dipole moments of $\mathrm{HOC}^{+}$and $\mathrm{HCO}^{+}$as $2.8 \mathrm{D}$ and $3.93 \mathrm{D}$, respectively, a slight departure from prior work in which we used 4.07D for $\mathrm{HCO}^{+}$. Given these assumptions, one has $N(\mathrm{X}) / \int \tau_{10} \mathrm{~d} v=2.146 \times 10^{12} \mathrm{~cm}^{-2}$ for $\mathrm{X}=\mathrm{HOC}^{+}$, $1.093 \times 10^{12} \mathrm{~cm}^{-2}$ for $\mathrm{X}=\mathrm{HCO}^{+}$and $1.128 \times 10^{12} \mathrm{~cm}^{-2}$ for $\mathrm{X}=\mathrm{H}^{13} \mathrm{CO}^{+}$.

\section{Observed and expected abundances of $\mathrm{HOC}^{+}$ in diffuse gas}

\subsection{Observed abundance of $\mathrm{HOC}^{+}$}

The mm-wave absorption data are summarized in Tables 1-3 and line profiles toward two sources are shown in Figs. 1 and 2. The $\mathrm{HCO}^{+}$and $\mathrm{OH}$ column densities are taken from our prior published (Lucas \& Liszt 1996; Liszt \& Lucas 1996) and unpublished work; for B0415+379 (see Fig. 1) we assert $N\left(\mathrm{HCO}^{+}\right)=59 \pm 2 N\left(\mathrm{H}^{13} \mathrm{CO}^{+}\right)($Lucas \& Liszt 1998) because of the high optical optical depth in the main isotope. $\mathrm{HOC}^{+}$ is found to have relative abundances $\mathrm{HCO}^{+}: \mathrm{HOC}^{+}=70-120$, $\mathrm{HOC}^{+}: \mathrm{OH}=3-6 \times 10^{-4}$ toward three sources (see Table 3 ). The results obtained toward the other two, B0528 and B1730,

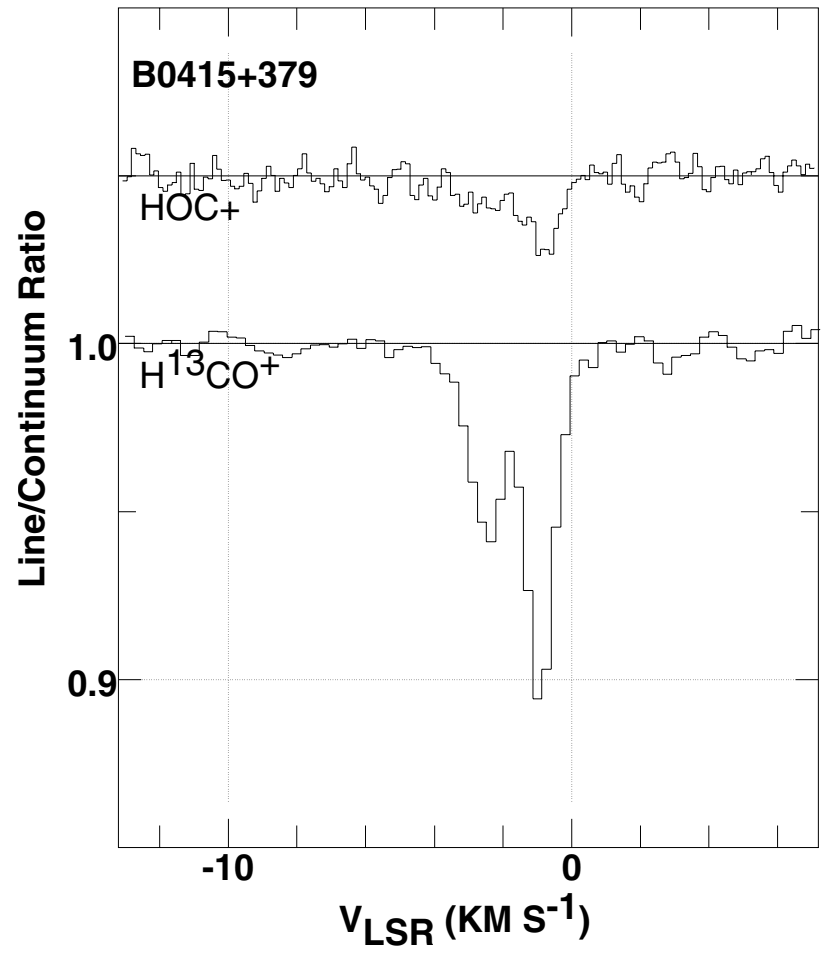

Fig. 1. $\mathrm{HOC}^{+}$and $\mathrm{H}^{13} \mathrm{CO}^{+}$observed toward B0415+379 (aka 3C 111).

Table 3. Column density ratios ${ }^{1}$.

\begin{tabular}{lcc}
\hline \hline Source & $N\left(\mathrm{HCO}^{+}\right) / N\left(\mathrm{HOC}^{+}\right)$ & $N\left(\mathrm{HOC}^{+}\right) / N(\mathrm{OH})$ \\
\hline B0355+508 & $72(9)$ & $6.1(0.7) \times 10^{-4}$ \\
B0415+379 & $117(12)$ & $2.6(0.3) \times 10^{-4}$ \\
B0528+134 & $>30$ & $<1.6 \times 10^{-3}$ \\
B1730-130 & $>18$ & $<4.3 \times 10^{-3}$ \\
B2200+420 & $70(24)$ & $6.5(2.1) \times 10^{-4}$ \\
\hline
\end{tabular}

${ }^{1}$ Limits are $2 \sigma$.

are not of great significance. The relative abundance of $\mathrm{OH}$, for which it is found that $X(\mathrm{OH})=n(\mathrm{OH}) / n\left(\mathrm{H}_{2}\right)=1.0 \pm 0.2 \times 10^{-7}$ along the four sightlines where both $N\left(\mathrm{H}_{2}\right)$ and $N(\mathrm{OH})$ have been measured (Liszt \& Lucas 2002), sets the relative abundance scale for $\mathrm{HOC}^{+}, X\left(\mathrm{HOC}^{+}\right)=3-6 \times 10^{-11}$.

Note that the $\mathrm{OH}$ column density toward $\mathrm{B} 2200+420$ is only $20 \%$ larger than that seen along the archetypical diffuse line of sight toward $\zeta$ Oph, $N(\mathrm{OH})=4.7 \pm 0.7 \times 10^{13} \mathrm{~cm}^{-2}$ (Van Dishoeck \& Black 1986; Roueff 1996), while the reddening $\left(E_{B-V}=0.33\right.$ mag toward B2200+420 from Schlegel et al. (1998)) is the same. The larger total $\mathrm{OH}$ column density seen toward $\mathrm{B} 0355+508$ at low galactic latitude $\left(-1.6^{\circ}\right)$ is roughly evenly divided among 5 kinematically-separated diffuse clouds of modest $\mathrm{OH}$ and $\mathrm{HCO}^{+}$column density, only two of which have prominent CO emission (Lucas \& Liszt 1996; Liszt \& Lucas 1996). The line of sight toward B0415+379 is somewhat darker (see the discussion in Lucas \& Liszt (1998)) but relatively little of the carbon along even this line of sight is in $\mathrm{CO}$ and the density is fairly low, as gauged by the weakness of emission from species beside CO (Lucas \& Liszt 1996). 


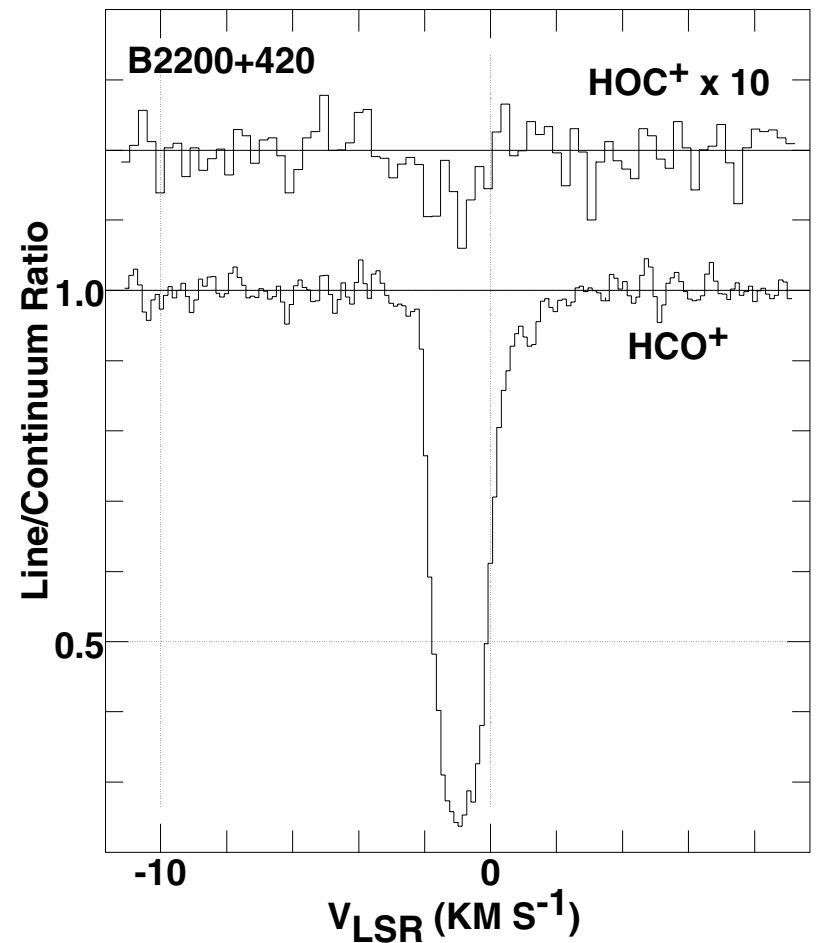

Fig. 2. $\mathrm{HOC}^{+}$and $\mathrm{HCO}^{+}$observed toward B2200+420 (aka BL Lac).

\subsection{Expected abundance of $\mathrm{HOC}^{+}$}

The most obvious path to $\mathrm{HOC}^{+}$in diffuse gas is via the reaction $\mathrm{C}^{+}+\mathrm{H}_{2} \mathrm{O} \rightarrow \mathrm{HOC}^{+}+\mathrm{H}$, which proceeds with a rate constant $1.8 \times 10^{-9} \mathrm{~cm}^{3} \mathrm{~s}^{-1}$ in the UMIST reaction database ${ }^{1}$ : these two reactants also produce $\mathrm{HCO}^{+}$at half the rate, but this is not an important source of $\mathrm{HCO}^{+}$. We parametrize the unknown abundance of water relative to $\mathrm{OH}$ and assume that all free carbon is once ionized in a fully molecular gas having a free carbon abundance $N(\mathrm{C}) / N(\mathrm{H})=1.4 \times 10^{-4}$ (Savage \& Sembach 1996). In these terms, the volume formation rate of $\mathrm{HOC}^{+}$from the reaction of $\mathrm{C}^{+}$and water can be expressed as $\mathrm{d} n\left(\mathrm{HOC}^{+}\right) / \mathrm{d} t=5.04 \times 10^{-20} n\left(\mathrm{H}_{2}\right)^{2} X\left(\mathrm{H}_{2} \mathbf{O}\right) / X(\mathrm{OH}) \mathrm{cm}^{-3} \mathrm{~s}^{-1}$.

Both $\mathrm{HCO}^{+}$and $\mathrm{HOC}^{+}$are formed from the reaction of $\mathrm{H}_{3}^{+}+\mathrm{CO} \rightarrow\left(\mathrm{HCO}^{+}\right.$or $\left.\mathrm{HOC}^{+}\right)+\mathrm{H}_{2}$. The $\mathrm{HCO}^{+} / \mathrm{HOC}^{+}$ branching ratio is typically taken as 20 (Illies et al. 1982) or more (the current UMIST reaction database has a branching ratio of 62). The volume formation rate from this reaction may be written as $\mathrm{d} n\left(\mathrm{HOC}^{+}\right) / \mathrm{d} t=2.7 \times 10^{-23} n\left(\mathrm{H}_{2}\right)^{2}$ $\left(X\left(\mathrm{H}_{3}^{+}\right) X(\mathrm{CO}) / 10^{-12}\right) \mathrm{cm}^{-3} \mathrm{~s}^{-1}$. The relative abundance of $\mathrm{CO}$ is known along these lines of sight to be $1-5 \%$ of the free carbon abundance (Liszt \& Lucas 1998), i.e. $1-5 \times 10^{-6}$. The observed amounts of $\mathrm{H}_{3}^{+}$, while surprisingly large (McCall et al. 2003) nevertheless still yield $X\left(\mathrm{H}_{3}^{+}\right)<10^{-6}$. Thus the only circumstance in which the reaction of $\mathrm{H}_{3}^{+}$and $\mathrm{CO}$ could be competitive in forming $\mathrm{HOC}^{+}$would be for very small water abundances, in which case neither formation route would suffice to form the observed $\mathrm{HOC}^{+}$.

\footnotetext{
${ }^{1}$ available at http://www.rate99.co.uk/
}

$\mathrm{HOC}^{+}$is likely destroyed by two processes which proceed with very nearly equal rates in diffuse gas. The $\mathrm{HOC}^{+}$isomer lies some $17000 \mathrm{~K}$ higher in energy compared to $\mathrm{HCO}^{+}$ and interconversion by molecular hydrogen $\left(\mathrm{HOC}^{+}+\mathrm{H}_{2} \rightarrow\right.$ $\mathrm{HOC}^{+}+\mathrm{H}_{2}$ ) has recently been shown to proceed with a rate constant $k_{I}=4 \times 10^{-10} \mathrm{~cm}^{3} \mathrm{~s}^{-1}$ nearly independent of temperature between $25 \mathrm{~K}$ and $300 \mathrm{~K}$ (Smith et al. 2002). This is $70 \%$ smaller than in the UMIST database, enhancing the prospects for reproducing $X\left(\mathrm{HOC}^{+}\right)$. The destruction rate for isomerization can be written as $\mathrm{d} n\left(\mathrm{HOC}^{+}\right) / \mathrm{d} t=-4 \times 10^{-10} n\left(\mathrm{H}_{2}\right)$ $n\left(\mathrm{HOC}^{+}\right) \mathrm{cm}^{-3} \mathrm{~s}^{-1}$.

Dissociative recombination, $\mathrm{HOC}^{+}+\mathrm{e}^{-} \rightarrow \mathrm{CO}+\mathrm{H}$, proceeds with a rate constant $1.1 \times 10^{-6}\left(30 / T_{\mathrm{K}}\right) \mathrm{cm}^{3} \mathrm{~s}^{-1}$. Assuming that free carbon is once-ionized and expressing the electron fraction in terms of the ratio of free carbon to $\mathrm{H}_{2}$ expected for a fully molecular diffuse gas (i.e. $N(\mathrm{C}) / N\left(\mathrm{H}_{2}\right)=$ $2 \times 1.4 \times 10^{-4}$ (Savage \& Sembach 1996)). The destruction rate from recombination can be written as $\mathrm{d} n\left(\mathrm{HOC}^{+}\right) / \mathrm{d} t=$ $-3.1 \times 10^{-10} n\left(\mathrm{H}_{2}\right) n\left(\mathrm{HOC}^{+}\right)\left(30 / T_{\mathrm{K}}\right)\left(X(\mathrm{e}) / X\left(\mathrm{C}^{+}\right)\right) \mathrm{cm}^{-3} \mathrm{~s}^{-1}$. The expected electron abundance is a small multiple of the free carbon abundance (Liszt 2003) so dissociative recombination and interconversion probably compete quite effectively to destroy $\mathrm{HOC}^{+}$.

The net destruction rate from both processes is of order $-10^{-9} n\left(\mathrm{H}_{2}\right) \mathrm{cm}^{-3} \mathrm{~s}^{-1}$ and the expected abundance of $\mathrm{HOC}^{+}$is $X\left(\mathrm{HOC}^{+}\right)=5 \times 10^{-11} X\left(\mathrm{H}_{2} \mathrm{O}\right) / X(\mathrm{OH})$ or $X\left(\mathrm{HOC}^{+}\right) / X(\mathrm{OH})=$ $5 \times 10^{-4} X\left(\mathrm{H}_{2} \mathrm{O}\right) / X(\mathrm{OH})$. The observed amounts of $\mathrm{HOC}^{+}$can be made in diffuse gas if the abundance of water is comparable to that of $\mathrm{OH}$. Limits on the $\mathrm{OH} /$ water ratio in diffuse clouds $N(\mathrm{OH}) / N\left(\mathrm{H}_{2} \mathrm{O}\right)>2-3$ were derived by Van Dishoeck \& Black (1986) toward $\zeta$ Oph and $\zeta$ Per.

Given the weakness of the limits on the water abundance in diffuse clouds, straightforward gas-phase chemistry fails only weakly to produce the observed amount of $\mathrm{HOC}^{+}$. This situation can be compared to the case of $\mathrm{HCO}^{+}$where the thermal reaction of $\mathrm{C}^{+}$upon the known quantities of $\mathrm{OH}$ falls 30-50 times short of producing the observed values of $\mathrm{N}\left(\mathrm{HCO}^{+}\right)$via the reaction chain $\mathrm{C}^{+}+\mathrm{OH} \rightarrow \mathrm{CO}^{+}+\mathrm{H}, \mathrm{H}_{2}+$ $\mathrm{CO}^{+} \rightarrow \mathrm{HCO}^{+}+\mathrm{H}, \mathrm{HCO}^{+}+\mathrm{e}^{-} \rightarrow \mathrm{CO}+\mathrm{H}$. At thermal rates, this series of reactions (which predicts an $\mathrm{HCO}^{+} / \mathrm{OH}$ ratio dependent only on temperature) produces $\mathrm{N}\left(\mathrm{HCO}^{+}\right)$scarcely larger than the observed $\mathrm{N}\left(\mathrm{HOC}^{+}\right)$. The predicted abundance of $\mathrm{HCO}^{+}$could be increased in quiescent gas by hypothesizing that it recombines slowly, but only at the cost of understanding CO (Liszt \& Lucas 2000).

Clearly, knowledge of the abundance of water is the limiting factor in assessment of the diffuse cloud chemistry of $\mathrm{HOC}^{+}$.

\subsection{Comparison with $\mathrm{HCO}^{+}$and $\mathrm{HOC}^{+}$in dense dark and bright gas}

The work presented here is the first evidence of $\mathrm{HOC}^{+}$in diffuse gas, but even the case for its existence in the dense molecular ISM was marginal before the pioneering work of Apponi and his collaborators during a brief period in the mid1990's while the NRAO 12 m telescope was still operating. 
To summarize the prior results we note that the $\mathrm{HCO}^{+} / \mathrm{HOC}^{+}$ ratio in dense dark gas is typically as large as 1000-9000 (Ziurys \& Apponi 1995; Apponi \& Ziurys 1997), but is found to be much smaller (50-400) in dense PDR like the Orion Bar (Apponi et al. 1999) and/or NGC 7023 (Fuente et al. 2003). In these PDR the relative abundance of $\mathrm{HOC}^{+}$is $X\left(\mathrm{HOC}^{+}\right)=$ $1-3 \times 10^{-11}$, quite similar to the diffuse clouds studied here.

The difference between the $\mathrm{HCO}^{+} / \mathrm{HOC}^{+}$ratios seen in diffuse and dark dense gas is largely the result of the low ionization fraction in the latter. Whereas $\mathrm{HOC}^{+}$is destroyed only slightly faster than $\mathrm{HCO}^{+}$in diffuse gas, the isomerization reaction undergone by $\mathrm{HOC}^{+}$is much faster than recombination when the electron fraction is $10^{-7}$ instead of $10^{-4}$. In dense dark gas $\mathrm{HCO}^{+}$and $\mathrm{HCO}^{+}$are formed by $\mathrm{H}_{3}^{+}+\mathrm{CO} \rightarrow$ $\mathrm{H}_{2}+\left(\mathrm{HCO}^{+}\right.$or $\left.\mathrm{HOC}^{+}\right)$with a branching ratio 20-60:1 in favor of $\mathrm{HCO}^{+}$and this ratio is increased by another factor of 100 by the unequal destruction rates. Under favorable conditions, measured $\mathrm{HCO}^{+} / \mathrm{HOC}^{+}$ratios could be good diagnostics of the ionization fraction in dense gas.

Reasoning by analogy from the discussion of diffuse and dark gas, we infer that the relatively low $\mathrm{HCO}^{+} / \mathrm{HOC}^{+}$ratios in dense PDR also reflect the relative weakness of isomerization. In cases where isomerization is weak both isomers are destroyed by recombination at roughly equal rates, and their abundance ratio is more directly indicative of the relative rates at which the two isomers are formed.

\section{Summary}

We have added $\mathrm{HOC}^{+}$to the roster of polyatomic molecules known to exist in diffuse clouds. The $\mathrm{HCO}^{+} / \mathrm{HOC}^{+}$ratio, $70-120$, is much smaller than in dense dark clouds but quite comparable to the most extreme values seen in dense PDR. The inferred relative abundance $X\left(\mathrm{HOC}^{+}\right)=3-6 \times 10^{-11}$, similar to that seen in dense PDR, can be synthesized at thermal rates in quiescent diffuse gas if the water/OH ratio is of order unity. This is a mild discrepancy, much less severe than is inferred for $\mathrm{HCO}^{+}$, as the water/OH ratio is currently known only to be less than $0.3-0.5$.

Several authors have recently noted that hydrocarbons like $\mathrm{C}_{3} \mathrm{H}_{2}$ and $\mathrm{C}_{2} \mathrm{H}$ have unexpectedly high abundances in dense PDR (Fosse et al. 2002; Fuente et al. 2003; Teyssier et al. 2004), very much as has been observed in diffuse clouds (Lucas \& Liszt 2000). The $\mathrm{HCO}^{+} / \mathrm{HOC}^{+}$ratios found here in diffuse clouds, which are PDR of lower density and presumably weaker illumination (hence similar ionization rates per $\mathrm{H}_{2}$ ), constitute another example of chemical similarities between dense and diffuse PDR. It will be interesting to see how much more complete a parallel can be drawn between the two regimes.

Acknowledgements. The National Radio Astronomy Observatory is operated by AUI, Inc. under a cooperative agreement with the US National Science Foundation. IRAM is operated by CNRS (France), the MPG (Germany) and the IGN (Spain). We owe the staff the Plateau de Bure our thanks for their assistance in taking the data. We thank the referee for helpful comments.

\section{References}

Apponi, A. J., Pesch, T. C., \& Ziurys, L. M. 1999, ApJ, 519, L89 Apponi, A. J., \& Ziurys, L. M. 1997, ApJ, 481, 800

Cox, P., Guesten, R., \& Henkel, C. 1988, A\&A, 206, 108

Federman, S. R., \& Willson, R. F. 1982, ApJ, 260, 124

Fosse, D., Teyssier, D., Gerin, M., et al. 2002, in Chemistry as a Diagnostic of Star Formation, 104

Fuente, A., Rodrıguez-Franco, A., Garcia-Burillo, S., Martın-Pintado, J., \& Black, J. H. 2003, A\&A, 406, 899

Illies, A. J., Jarrold, M. F., \& Bowers, M. T. 1982, J. Chem. Phys., 77, 5847

Liszt, H. 2003, A\&A, 398, 621

Liszt, H., \& Lucas, R. 2001, A\&A, 370, 576

Liszt, H., \& Lucas, R. 2002, A\&A, 391, 693

Liszt, H. S., \& Lucas, R. 1995, A\&A, 299, 847

Liszt, H. S., \& Lucas, R. 1996, A\&A, 314, 917

Liszt, H. S., \& Lucas, R. 1998, A\&A, 339, 561

Liszt, H. S., \& Lucas, R. 2000, A\&A, 355, 333

Lucas, R., \& Liszt, H. 1998, A\&A, 337, 246

Lucas, R., \& Liszt, H. S. 1996, A\&A, 307, 237

Lucas, R., \& Liszt, H. S. 2000, A\&A, 358, 1069

Lucas, R., \& Liszt, H. S. 2002, A\&A, 384, 1054

Maier, J. P., Lakin, N. M., Walker, G. A. H., \& Bohlender, D. A. 2001, ApJ, 553, 267

Marscher, A. P., Moore, E. M., \& Bania, T. M. 1993, ApJ, 419, L101

McCall, B. J., Huneycutt, A. J., Saykally, R. J., et al. 2003, Nature, 422, 500

Moore, E. M., \& Marscher, A. P. 1995, ApJ, 452, 671

Nash, A. G. 1990, Astrophys. J., Suppl. Ser., 72, 303

Oka, T., Thorburn, J. A., McCall, B. J., et al. 2003, ApJ, 582, 823

Roueff, E. 1996, MNRAS, 279, L37

Roueff, E., Felenbok, P., Black, J. H., \& Gry, C. 2002, A\&A, 384, 629

Savage, B. D., \& Sembach, K. R. 1996, ARA\&A, 34, 279

Schlegel, D. J., Finkbeiner, D. P., \& Davis, M. 1998, ApJ, 500, 525

Smith, M. A., Schlemmer, S., von Richthofen, J., \& Gerlich, D. 2002, ApJ, 578, L87

Teyssier, D., Fossé, D., Gerin, M., et al. 2004, A\&A, 417, 135

Van Dishoeck, E. F., \& Black, J. H. 1986, ApJS, 62, 109

Ziurys, L. M., \& Apponi, A. J. 1995, ApJ, 455, L73 